\title{
Multi-timescale Collaborative Optimization of Distribution, Distributed Generation and Load in Microgrid
}

\author{
Wen Hu, Yun-lian Sun, Yang Wang, Yang-jun Zhou, Meng-ying Wang \\ School of Electrical Engineering, Wuhan University, Wuhan City, Hubei Province, China \\ Email: huwen@whu.edu.cn
}

Received 2013

\begin{abstract}
The distribution loads, output of distributed generations (DGs) and dynamic power price present obvious time-sequence property, the typical property is studied in this paper. The model of microgrid (including adjustable load, DGs, storage and dynamic power price) is studied. A multi-timescale collaborative optimization model is built towards microgrid; main measures in different timescale optimization are realized. An improved adaptive genetic algorithm is used to solve the optimization problem, which improved the efficiency and reliability. The proposed optimization model is simulated in IEEE 33 node system; the results show it's effective.
\end{abstract}

Keywords: Microgrid; Multi-timescale; Collaborative Optimization; Time-sequence Property; Improved Adaptive Genetic Algorithm (IAGA)

\section{Introduction}

The smart grid is not exactly same as a microgrid; they could offer two competing visions of bringing technological innovation to the electricity grids. The goals of both are same: to maximize services provided by generation and storage through embedded intelligence, while dramatically boosting efficiencies and minimizing costs [1].

The microgrid, a typical one as shown in Figure 1, can be summed up as follows [2]: an integrated energy system network consisting of distributed generations (DGs) and electrical loads and/or meters operating as a autonomous grid either in parallel to or islanded from the utility grid. DGs include photovoltaic power (PV), wind turbine (WT), electrochemical cell, marsh gas generation and so forth; are generally tied together on their own feeder, which is linked to the utility grid at a point of common coupling (PCC).

A microgrid is a small-scale version of the traditional electricity grid. Like traditional power grid, microgrids include generation facilities, distribution lines, and voltage regulators. They can be networked with others to boost capacity, efficiency, and reliability - or can function as autonomous islands of power during times of emergency or to respond to real-time market conditions.

This paper brought a multi-timescale model of collaborative optimization, for the essential factors in microgrid present obvious time-sequence property. Genetic algorithm is improved by adaptive parameters, to solve the optimization problem. The simulations reveal that both the optimization model and algorithm are efficient.

\section{Collaborative Optimization in Microgrid}

Collaborative optimization of distribution needs the coordination of distributed generation (DG) output, adjustable load and distribution operation. The optimization leads to the optimal and economical operation of distribution network, including:

- Improving the efficiency of the distribution network.

- Improve the power quality.

- Promoting the admitting ability of DG supply.

- Improving the utilization results of DG.

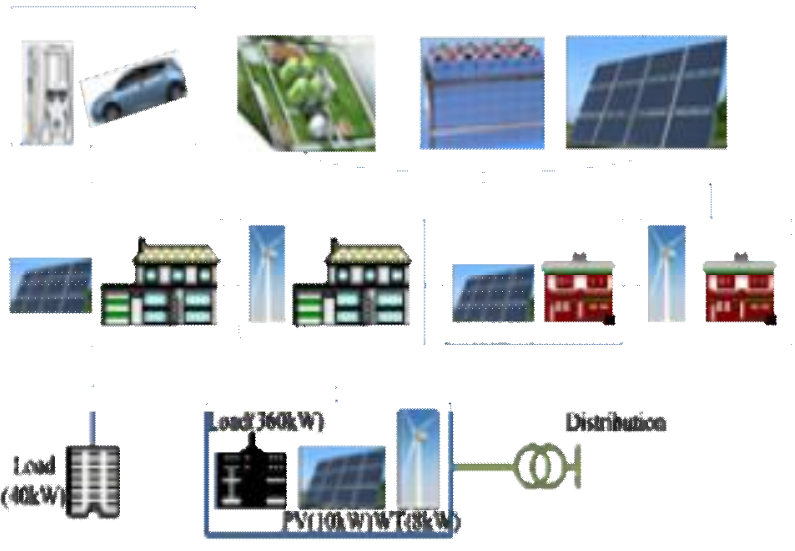

Figure 1. Microgrid topology. 
Power system network structure, load characteristic and output characteristics of DG are influential to the operating state of grid. The uncertainty, randomness and volatility of these subject, are time-varying. Hence, the model of collaborative optimization is established in different time scale, shows in Figure 2.

\subsection{Long Term Optimization}

The power loss and reliability are two main economic and technical targets to evaluate the distribution. Thus, the optimization model is built based on both:

$$
\min F_{1}=\eta_{1} \sum_{n=1}^{N_{F}} \omega_{n} f_{1}+\eta_{2} \sum_{n=1}^{N_{F}} \omega_{n} f_{2}
$$

In formula above: $\eta_{1}$ and $\eta_{2}$ are the weight of power loss and reliability, $\omega_{n}$ is the weight of scenario n, $N_{F}$ is the number of scenario formed by the time-sequence property of DGs output and loads.

The mathematical model of power $\operatorname{loss} f_{1}$ is:

$$
f_{1}=\sum_{i=1}^{N_{l}} k_{i} R_{i} \frac{P_{i}^{2}+Q_{i}^{2}}{\left|V_{i}\right|^{2}}
$$

In formula above: $P_{i}$ and $Q_{i}$ are the active power and reactive power which flow through the branch $i ; V_{i}$ is the voltage at the end of the branch $i ; k_{i}$ is the state variable of branch $i, 0$ means that branch is out of the power supply, 1 means that branch is at the power supply; $R_{i}$ is the resistance of branch $i ; N_{l}$ is the number of the branches.

The mathematical model of reliability $f_{2}$ is:

$$
f_{2}=\sum_{i=1}^{N u m} \sum_{j=1}^{N} \lambda_{j} \gamma_{j} P_{i j}
$$

In formula above: $\lambda_{j}$ is the failure rate of load $j, \gamma_{j}$ is the average interruption duration index of load $\mathrm{j}, P_{i j}$ is the size of load $j$ at time $i$.

Subject to:

Subject to

1) Voltage constraints

$$
V_{j \min } \leq V_{j} \leq V_{j \max }
$$

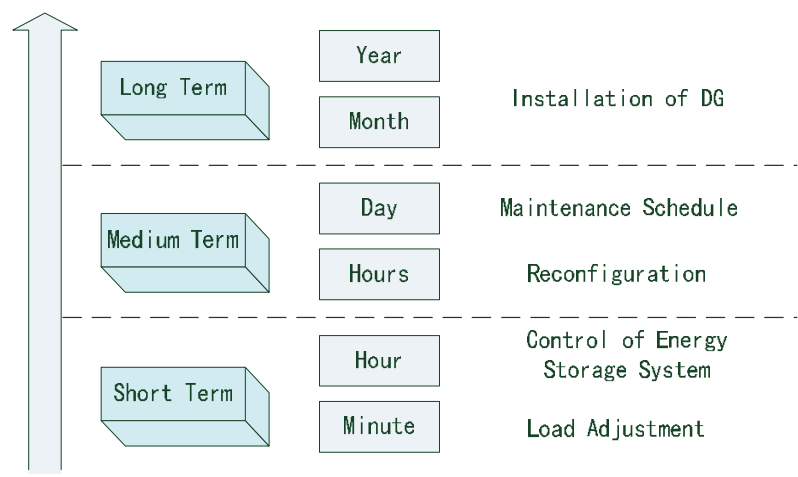

Figure 2. Multi-timescale collaborative optimization.
2) Branch power constraints

$$
S_{j} \leq S_{j \max }
$$

3) Flow constraints

$$
A X=D
$$

4) Power supply constraints: Network can't have island nodes and loops

In formula above: $V_{j \min }$ and $V_{\text {jmax }}$ are the minimum and maximum of the voltage of the node $j ; S_{\text {jmax }}$ is the maximum transmission power value of the branch $j$; A is network associated matrix; $\mathrm{X}$ is the branch flow vector of the branch; $\mathrm{D}$ is load vector.

\subsection{Medium Term Optimization}

Optimization reconfiguration is the main method of medium term optimization, its main target is divided into:

- Reduce the risk of load shedding

- Minimize fault recovery time and outage range

- Even distributed loads, to avoid overload

- Minimize the power loss

- Minimize the ullage of energy in given time

The mathematical model of recovery reconfiguration in this paper is:

$$
\min F_{2}=f_{1}
$$

Subject to the same condition as above.

\subsection{Short Term Optimization}

The short term optimization is based on the DR-VPP parameter, which serves the grid in a dynamic, real-time manner. Battery and adjustable load are considered as DR-VPP parameter. Its pros include:

- DR-VPPs represent a new wave business model, for they are highly dependent on utility investments in smart meters and advanced metering infrastructure (AMI) infrastructure.

- VPPs represent an open and highly scalable program, are designed to accommodate the unique characteristics of an end user's environment.

- DR is a core of the smart grid movement, will turn a wide range of formerly passive energy consumers into active energy market participants.

- Since the DR is based on software and IT innovations, it can be used widely and deployed across broad sectors and geographic locations, incorporating a variety of DGs and energy storage.

VPPs are really an alternative approach to the aggregation of DG, thus, may be considered an alternative or complement to the microgrid. VPPs are envisioned to tap supply, demand, and energy storage devices. With its emphasis on smart meters, dynamic pricing, and DR, the smart grid is actually a prerequisite for VPPs. Electric power demand side response influences the demand time 
and level based on the system reliability programs or market prices. Load reductions aggregated from grid-tied microgrids can be sold into power markets as three distinct products [3]:

- Peak capacity products to help maintain a utility's $15 \%$ supply reserve margin

- Economic energy, which can be sold on an hour-by-hour basis

- Grid regulation services, which can last for a matter of minutes

Federal Energy Regulatory Commission (FERC) estimates that DR can achieve a reduction of peak demand of $4 \%$ to $9 \%$, depending on the penetration of DR technology, the number of participants, and the dynamic pricing structures chosen.

\section{Time-sequence Property}

With the time-sequence property of load and DG output considered, the full process simulation makes the optimization results approximate to the actual, and main economic and technical targets of distribution are faithfully represented. Besides, electrovalence has time-sequence property after realizing demand side response and electricity market reformation. The basic characteristics are as follows:

- All objects (except of TOU power price) are timevarying, but the time-sequence property follows the regular pattern that applies to the weather, season, and time of day.

- The maximal load and DG output always happens at different time, thus, the dynamic power price is uncertain.

- The WT and PV have a time-sequence property of complementary.

- Sequential process simulation of all objects is needed to represent the technical index of the microgrid and distribution.

\subsection{Time-sequence Property of Load}

Electrical load is divided into four typical types: industry, agriculture, commerce, and municipal life, which industrial features varies. Figure 3 shows the time-sequence property of four types of load, light industry is studied [4].

Short-term load forecasting usually use a normal distribution to represent the non-determinacy of load. The active load and reactive load of node $i$ at time $t$ is:

$P_{L, i, t} \sim N\left(\mu_{P L, i, t}, \sigma_{P L, i, t}^{2}\right)$, and $Q_{L, i, t} \sim N\left(\mu_{Q L, i, t}, \sigma_{Q L, i, t}^{2}\right) \cdot \mu_{\mathrm{PL}, \mathrm{i}, t}$, $\mu_{Q L, i, t}, \sigma_{\mathrm{PL}, \mathrm{i}, \mathrm{t}}^{2}$, and $\sigma_{Q L, i, t}^{2}$ are the mean value and mean square error of $P_{L, i, t}$ and $Q_{L, i, t}$.

\subsection{Time-sequence Property of DG Output}

Output of DG is mainly determined by geographical lo- cation and climatic environment, the typical time-sequence property of which is shown in Figure 4.

The property of WT has direct relation with wind resources. The daily variation of wind speed, divide into nautical and terrestrial type, varies a lot in different season. The property of PV has direct relation with illumination intensity, which is direct influenced by weather and season. The illumination intensity curves can be divided into 3 types in the same season: sunshine, rainy day and overcast day.

The short-time forecasting of wind speed uses Weibull distribution, shown in formula (5). The output of PV is determined by illumination intensity, temperature and humidity. Illumination intensity is considered only for simplify, the short-time forecasting of it uses Beta distribution, shown in formula (6)[5].

$$
f(v)=\frac{k}{c} \cdot\left(\frac{v}{c}\right)^{k-1} \cdot \exp \left[-\left(\frac{v}{c}\right)^{k}\right]
$$

In formula above: $v$ is wind speed, $k$ and $c$ are the shape parameter and scale parameter of Weibull distribution, $k=(\sigma / \mu)^{-1.086}, c=\mu / \Gamma(1+1 / k), \mu$ is average wind velocity, $\sigma$ is standard deviation, $\Gamma$ is Gamma function.

$$
f(r)=\frac{\Gamma(\alpha+\beta)}{\Gamma(\alpha) \Gamma(\beta)}\left(\frac{r}{r_{\max }}\right)^{\alpha-1}\left(1-\frac{r}{r_{\max }}\right)^{\beta-1}
$$

In formula above: $r$ and $r_{\max }$ are the actual light and maximum illumination intensity in the period, $\alpha$ and $\beta$

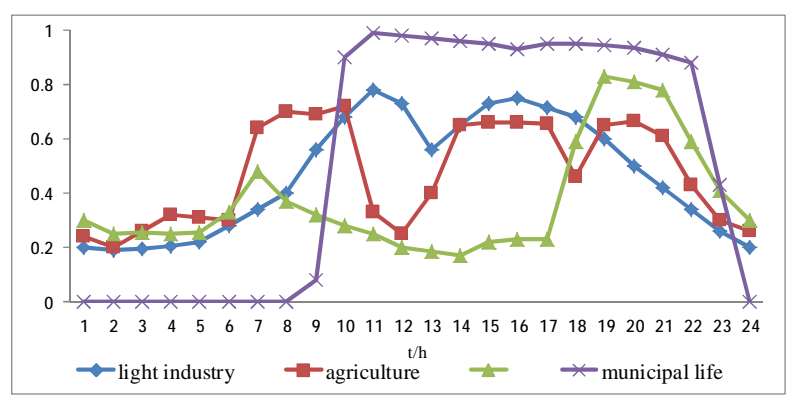

Figure 3. Time-sequence property curves of four types of load.

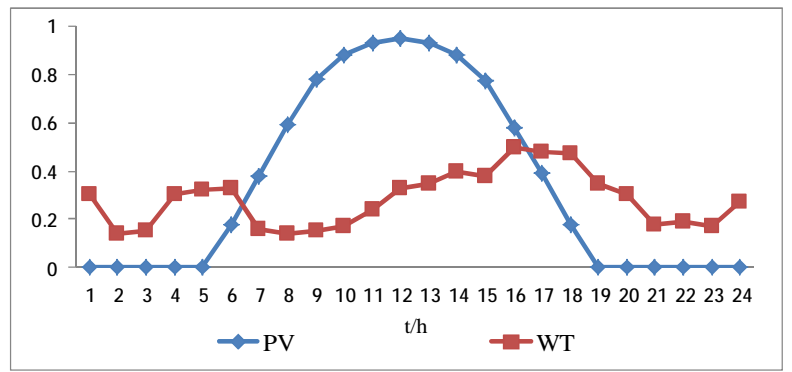

Figure 4. Time-sequence property curves of daily power output of DG. 
are the shape parameter of Beta distribution which can get by the average value $\mu$ and variance $\sigma$ of illumination intensity.

$$
\begin{gathered}
\alpha=\mu\left[\frac{\mu(1-\mu)}{\sigma^{2}}-1\right] \\
\beta=(1-\mu)\left[\frac{\mu(1-\mu)}{\sigma^{2}}-1\right]
\end{gathered}
$$

\subsection{Time-sequence Property of Electrovalence}

Time-of-use (TOU) power price [6] and dynamic power price are important measures in demand side response. TOU power price can efficiently reflect electricity pricing mechanism of power supply cost in different timeinterval. Dynamic power price can accurately reflect the fluctuation of electricity pricing mechanism in every time-interval and realize the optimal allocation of power resources, by linking $\mathrm{t}$ market clearing price and sale price of retail side. A typical time-sequence property of both is shown in Figure 5.

\section{Improved Adaptive Genetic Algorithm}

Genetic algorithm (GA) is a kind of optimization algorithms, simulates Darwin's genetic choice and natural elimination biology evolution process. GA searches multiple point parameters of the code based on random transformation rules, existing the early convergence and slow convergence. To solve the problem, an improved adaptive genetic algorithm (IAGA) is used.

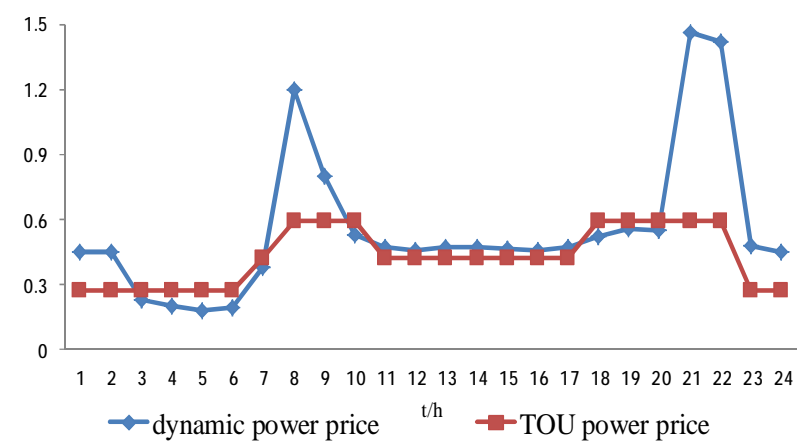

Figure 5. Time-sequence property curves of electrovalence.

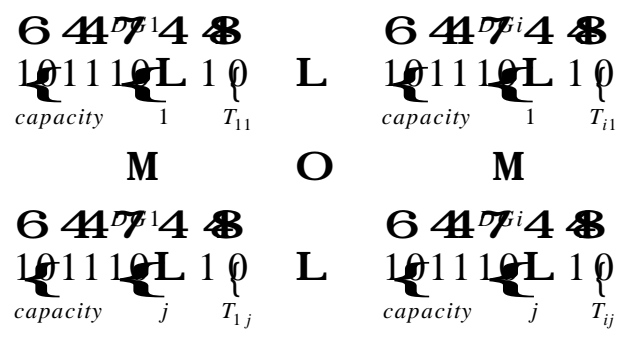

Figure 6. Chromosome structure.
The GA process includes: chromosome encode, the form of the initial population, fitness value evaluation, and genetic operation.

\subsection{Chromosome Encode}

Binary chromosome encoding is used, but varies in the 3 optimization.

1) Long term optimization

To locate and size the DGs, the segmented binary chromosome encoding is used: segments represent the number of DG types, each section of the code represent a location and size of a DG.

Chromosome, shown in Figure 6, has $m$ segment (means the number of DG types is $m$ ); section $i$ means the location and size of DG No. $i ; T_{i j}$ means the installation conditions of DG No. $i$ at node $j$; the capacity of DG is expressed with a 4 bits, which represents heterogeneous of capacity.

2) Medium term optimization

Binary coding based on the loop is used, to shorten the length of chromosome and avoid a large number of infeasible solutions:

- Coding of " 0 " indicates that the switch is open, Coding of " 1 " indicates that the switch is closed.

- The switch, connects with power source directly, should be closed.

3) Short term optimization

Similar with Figure 6, the chromosome has $m$ segment ( $m$ means the number of node with battery or adjustable load), $T_{i j}$ means the conditions of No. $i$ at node $j$ (" 1 " represents forward currents, " 0 " represents negative or no currents).

The form of the initial population in medium term optimization is based on loop.

\subsection{Genetic Operation}

The age and lifetime of individual [7] are used to avoid population size increasing too fast. The age increases with every genetic algebra. When age reaches the lifetime, the individual dies. Lifetime is calculated every generation:

$$
L T(t+1, x)=\left\{\begin{array}{l}
L T(t, x)-\theta \frac{F i t(t, x)-F i t_{W}}{F i t_{A}-F i t_{W}}, F i t(t, x) \leq F i t_{A} \\
L T(t, x)+\theta \frac{F i t(t, x)-F i t_{A}}{F i t_{B}-F i t_{A}}, F i t(t, x)-F i t_{A}
\end{array}\right.
$$

In formula above: $\theta=L T(t, x)$-age $(x) ; L T(t, x)$ and Fit $(t, x)$ are the age and lifetime of individual $x$ at generation $t ; \mathrm{Fit}_{B}, \mathrm{Fit}_{A}$ and $\mathrm{Fit}_{W}$ are the best, average, worst fitness of the individuals of present population; age $(x)$ is the age of individual $x$.

To macro-control the generation in case it being too 
huge to calculate [8], the mathematical model is:

$$
\begin{gathered}
P s(t+1) \\
=\left\{\begin{array}{c}
P s(t)+N P(t)-D P(t), \quad P s(t) \leq \varphi \\
P_{n 1} P s(t)+N P(t)-D P(t), \quad \varphi<P s(t) \leq 0.5 \Phi \\
P_{n 2} P s(t)+N P(t)-D P(t), \quad 0.5 \Phi<P s(t) \leq \Phi \\
\Phi-D P(t), P s(t)>\Phi
\end{array}\right.
\end{gathered}
$$

In formula above: $\operatorname{Ps}(\mathrm{t})$ is the population size; $\mathrm{NP}(\mathrm{t})$ is the new population formed by crossover and mutation operation; $\mathrm{DP}(\mathrm{t})$ is the number of individuals weeded out at generation $\mathrm{t}$; $\Phi$ and $\varphi$ are the maximal population size settled and initial size; Pn1 and $\mathrm{Pn} 2$ are reproduction ratio, $\mathrm{Pn} 1>\mathrm{Pn} 2$.

The crossover and mutation operation operates based on the optimization characteristic. In order to speed up the convergence, the crossover and mutation rates are adjusted adaptively according to the evolution situation. The mathematical model is:

$$
\begin{aligned}
& P_{c}=\left\{\begin{array}{l}
\frac{k_{1}\left[P s(t+1)-F i t_{A}\right]}{P s(t+1)-f_{A}}, f_{A} \mathrm{p} F i t_{A} \\
k_{3}, f_{A} \geq F i t_{A}
\end{array}\right. \\
& P_{m}=\left\{\begin{array}{l}
\frac{k_{2}\left[P s(t+1)-F i t_{A}\right]}{P s(t+1)-f_{A}}, f_{A} \mathrm{p} F i t_{A} \\
k_{4}, f_{A} \geq F i t_{A}
\end{array}\right.
\end{aligned}
$$

where, $P_{c}$ and $P_{m}$ are crossover and mutation rate; $f_{A}$ is the average fitness of the children population. $k_{1}, k_{2}, k_{3}$ and $k_{4}$ is constant, ranging in $[0,1], k_{3}>k_{1}, k_{4}>k_{2}$.

\subsection{The Terminal Criterion of the Algorithm}

The convergence degree of population and maximum evolution algebra are using as the terminal criterion of the algorithm.

\section{The Simulation Results and Analysis}

The example uses IEEE33 nodes distribution network system, which has 33 nodes and 37 branches. The system has 5 loops. The rated voltage and power are $12.66 \mathrm{kV}$ and 10MVA. Network parameters and nodes of load are in reference [9]. The initial population size is set as 60 . The initial crossover rate and mutation rate are 0.6 and $0.01, k_{1}, k_{2}, k_{3}, k_{4}$ are $0.5,0.05,0.6,0.1 . P n$ is 0.6 . When $t$ is $0, L T(t, x)$ is 50 . The maximum number of iterations is 100. The compared result of GA, improved GA [10] (IGA) and algorithm in this paper (IAGA) is shown in Table 1.

As shown in Table 1: using IGA and IAGA get better convergence results than GA, while IAGA play a better role than IGA in reconfiguration computing. The rational utilization of DG can reduce the loss obviously.
Table 1. Results of optimization.

\begin{tabular}{rcccc}
\hline & \multicolumn{4}{c}{ Loss (kW) } \\
\cline { 2 - 5 } & Initial & $\begin{array}{c}\text { DG } \\
\text { accessed }\end{array}$ & $\begin{array}{c}\text { Reconfiguration } \\
\text { without DG }\end{array}$ & $\begin{array}{c}\text { Reconfiguration } \\
\text { with DG }\end{array}$ \\
\hline GA & 202.7 & 102.2 & 163.9 & 102.2 \\
IGA & 202.7 & 82.4 & 142.6 & 82.4 \\
IAGA & 202.7 & 82.4 & 136.1 & 78.5 \\
\hline
\end{tabular}

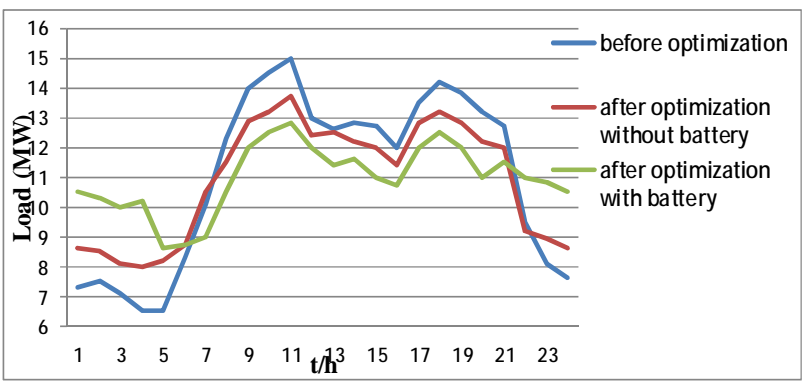

Figure 7. Load curves before and after the optimization.

Figure 7 shows the load curves before and the shortterm optimization. It is obvious that the valley-to-peak reduced $24.5 \%$ after the optimization, the profile could be better with more battery and adjustable loads.

\section{Conclusions}

This paper brought a multi-timescale model of collaborative optimization, for the time-sequence property factors in microgrid. With reasonable location and capacity, the long-term optimization can improve the technical efficiency. The medium-term optimization improves technical efficiency the network loss of microgrid through reconfiguration. The short-term optimization can reduce the valley-to-peak and peak load, and guide rational power consumption.

\section{REFERENCES}

[1] R. H. Lasseter, "Smart Distribution: Coupled Microgrids," Proceedings of the IEEE, Vol. 13, No. 8, 2011, pp. 1074-1082. doi:10.1109/JPROC.2011.2114630

[2] Y. X. Yu and W. P. Luan, "Smart Grid and Its Implementations," Proceedings of the CSEE, Vol. 29, No. 34, 2009, pp. 1-6.

[3] F. Bin, "Research of Electricity Price Regulation Method and Application," Ph. D Thesis, North China Electric Power University, Beijing, 2010.

[4] L. LI, W. Tang and M. K. Bai, "Multi-objective Location and Sizing of Distributed Generators based on Time-sequence Characteristics," Automation of Electric Power, Vol. 37, No. 3, 2013, pp. 58-63.

[5] G. Chen, P. Dai and H. Zhou "Distribution System Reconfiguration Considering Distributed Generators and Plug-in Electric Vehicles," Power System Technology, 
Vol. 37, No. 1, 2013, pp. 82-88.

[6] J. Li, J. Y. Liu, L. F. Xie, H. Quan and Y. B. Liu, "Dynamic Game Linkage of TOU Pricing Between Generating Side and Retail Side," Electric Power Automation Equipment, Vol. 32, No. 4, 2012, pp. 16-19.

[7] J. Arabas, Z. Michalewicz and J. Mulawka, "GAVaPS: A Genetic Algorithm with Varying Population Size," Proceedings of the 1st IEEE Conference on Evolutionary Computation, 1994, pp. 73-78.

[8] X. Jin, "The Application of Genetic Algorithm with
Adaptive Population Size in Distribution Network," North China Electric Power University, Beijing, 2011.

[9] H. Zhang, "Study of Distribution Network Fault Restoration Based on Genetic and Particle Swarm Mixed Algorithm," Wuhan University, Wuhan, 2009.

[10] S. Q. Sheng, Z. G. Ma and J. Wu, "Distribution Network Fault Restoration Based on Improved Adaptive Genetic Algorithm," Second Conference on Intelligent Computation Technology and Automation, 2009. 